\title{
Quantifying the latitudinal representivity of in situ solar wind observations
}

\author{
Mathew J. Owens ${ }^{1, *}$, Matthew Lang ${ }^{1}$, Pete Riley ${ }^{2}$, Mike Lockwood ${ }^{1}$, and Amos S. Lawless ${ }^{1,3}$ \\ ${ }^{1}$ Department of Meteorology, University of Reading, Earley Gate, PO Box 243, RG6 6BB Reading, UK \\ ${ }^{2}$ Predictive Science Inc., 9990 Mesa Rim Rd, Suite 170, San Diego, 92121 CA, USA \\ ${ }^{3}$ Department of Mathematics and Statistics, University of Reading, Whiteknights, PO Box 220, RG6 6AX Reading, UK
}

Received 18 September 2019 / Accepted 18 February 2020

\begin{abstract}
Advanced space-weather forecasting relies on the ability to accurately predict near-Earth solar wind conditions. For this purpose, physics-based, global numerical models of the solar wind are initialized with photospheric magnetic field and coronagraph observations, but no further observation constraints are imposed between the upper corona and Earth orbit. Data assimilation (DA) of the available in situ solar wind observations into the models could potentially provide additional constraints, improving solar wind reconstructions, and forecasts. However, in order to effectively combine the model and observations, it is necessary to quantify the error introduced by assuming point measurements are representative of the model state. In particular, the range of heliographic latitudes over which in situ solar wind speed measurements are representative is of primary importance, but particularly difficult to assess from observations alone. In this study we use 40+ years of observation-driven solar wind model results to assess two related properties: the latitudinal representivity error introduced by assuming the solar wind speed measured at a given latitude is the same as that at the heliographic equator, and the range of latitudes over which a solar wind measurement should influence the model state, referred to as the observational localisation. These values are quantified for future use in solar wind DA schemes as a function of solar cycle phase, measurement latitude, and error tolerance. In general, we find that in situ solar wind speed measurements near the ecliptic plane at solar minimum are extremely localised, being similar over only $1^{\circ}$ or $2^{\circ}$ of latitude. In the uniform polar fast wind above approximately $40^{\circ}$ latitude at solar minimum, the latitudinal representivity error drops. At solar maximum, the increased variability of the solar wind speed at high latitudes means that the latitudinal representivity error increases at the poles, though becomes greater in the ecliptic, as long as moderate speed errors can be tolerated. The heliospheric magnetic field and solar wind density and temperature show very similar behaviour.
\end{abstract}

Keywords: Solar wind / space weather forecasting / data assimilation

\section{Introduction}

Near-Earth solar wind conditions are routinely forecast for several days ahead on the basis of photospheric magnetic field observations (e.g., MacNeice et al., 2018). Such forecast schemes typically involve two coupled models; a steady-state coronal model (e.g., Linker et al., 1999; Arge et al., 2003) that covers the photosphere to approximately 20 solar radii $\left(\mathrm{R}_{\odot}\right)$, and a heliospheric model (e.g., Riley et al., 2001; Odstrcil, 2003; Toth et al., 2005; Pomoell \& Poedts, 2018) that spans approximately $20 \mathrm{R}_{\odot}$ to at least $215 \mathrm{R}_{\odot}(1 \mathrm{AU})$. Solar wind speed at the heliospheric inner-boundary is derived from

\footnotetext{
*Corresponding author: m. j. owens@reading. ac.uk
}

imperfect empirical relations to the coronal magnetic field configuration and consequently introduces a large degree of uncertainty (Arge et al., 2003; McGregor et al., 2011; Riley et al., 2015). Transient solar wind structures representing coronal mass ejections can be characterised using coronagraph observations and used to modify the inner boundary conditions to the heliospheric models (Zhao et al., 2002; Mays et al., 2015). But beyond this distance, no further observational constraints are placed on the heliospheric models, i.e. they are entirely "free running" from $20 \mathrm{R}_{\odot}$ to $215 \mathrm{R}_{\odot}$.

For both space-weather forecasting and reconstruction of past heliospheric conditions, it is desirable to constrain heliospheric models using all available observations. Data assimilation (DA) is the mathematically rigorous process of 
combining models and observations, taking account of their individual uncertainties (Kalnay, 2002; Asch et al., 2016). This methodology has long been used in atmospheric and oceanic sciences (Sasaki, 1970; Ghil \& Malanotte-Rizzoli, 1991; Rabier, 2005) and has more recently been adopted in relatively data-rich areas of space physics (Bust \& Mitchell, 2008; Hickmann et al., 2015; Glauert et al., 2018; Temmer et al., 2018; Aseev \& Shprits, 2019; Elvidge \& Angling, 2019). In the heliosphere, we have recently demonstrated assimilation of in situ solar wind observations (Lang et al., 2017; Lang \& Owens, 2019), though the same methods should be broadly applicable to other remote observing techniques in the future (e.g., Manoharan, 2012; Barnard et al., 2019). In order to accommodate in situ measurements into the model state, it is necessary to know the spatial domain over which measurements are representative and the error introduced by any spatial mismatch between model and measurement point (Waller et al., 2014; Janji et al., 2018). A stationary spacecraft will sample all solar longitudes over a complete solar rotation, which is equivalent to sampling all longitudes at a single time if the solar wind is assumed to be in a quasi-steady state (e.g., Kahler et al., 2016). This assumption is increasing invalid as solar activity, and the dynamic nature of the corona, increases. In the radial direction, observations can be mapped back or forwards by considering momentum exchange between solar wind streams. Thus under the steady-state assumption, observations can be adequately mapped in longitude and radial distance. The difficulty is knowing the heliographic latitude range over which a measurement is representative. For DA within a three-dimensional solar wind model (e.g., Lang et al., 2017), this is essential information in terms of the "localisation"; the volume of the model state that is relevant to - and thus should be influenced by - measurements from a given point in space. The assumed latitudinal range of influence can have a large effect on the reconstructed solar wind, even over the small heliographic latitude ranges sampled by in-ecliptic spacecraft (Owens et al., 2019b). For DA with a two-dimensional solar wind model (e.g., Lang \& Owens, 2019), a latitudinal representivity error is introduced by using in situ measurements from latitudes away from that being modelled (typically the heliographic equatorial plane). This, along with the inherent measurement error (small for in situ observations) forms part of the "observational error" in DA.

Computing these solar wind latitudinal representivity properties from current or planned observations is difficult, if not impossible; there are no pairs of observations at the same radial distances and longitudes, but at a range of latitudinal separations. Furthermore, the latitudinal representivity error is expected to be a strong function of solar activity (e.g., McComas et al., 2003), requiring any such data to be further stratified.

Recently, we used magnetogram-constrained simulations of the solar wind to estimate the difference in solar wind speed encountered at Earth and the L5 position, $60^{\circ}$ behind Earth in its orbit, owing entirely to the small time-varying difference in latitude (Owens et al., 2019a, hereafter "Paper 1"). While the latitudinal difference is small, varying from $0^{\circ}$ to approximately $7^{\circ}$ over the year, it can lead to sampling significantly different solar wind streams at the two locations, even under the perfectly steady-state approximation. This is a result of a systematic displacement relative to the heliospheric current sheet (HCS) and the associated solar wind speed structures.
Paper 1 showed that this difference is maximized at solar minimum and minimized at solar maximum, owing to the respective latitudinal structuring of the solar wind at those times. The focus of Paper 1 was on the limitations of L5 observations to provide a forecast of near-Earth conditions. In this study, we exploit the same dataset to quantify the latitudinal representivity properties as a function of latitude and solar activity. While no data assimilation is performed in this study, it seeks to estimate properties which are necessary to enable effective solar wind DA in future studies.

\section{Data and methods}

We use the same dataset detailed in Paper 1 and Owens et al. (2017). In summary, it comprises $40+$ years of solar wind simulations from the magnetohydrodynamics algorithm outside a sphere (MAS) global coronal and heliosphere model (Linker et al., 1999; Riley et al., 2012) constrained using the observed photospheric magnetic field. The heliospheric model covers 30-215 $\mathrm{R}_{\odot}$ and has 128,111 , and 140 gridcells in the azimuthal, meridional, and radial directions. Thus the gridcell size at $1 \mathrm{AU}$ means the model will not be able to resolve radial structures below $\sim 10^{6} \mathrm{~km}$ and time scales shorter than approximately $1 \mathrm{~h}$. The steady-state approximation of the solar wind solutions means that the values presented here represent a lower limit, with transient structures, small-scale turbulence, and rapidly-evolving large-scale structures providing additional sources of variability.

Examples of the obtained 1-AU solar wind solutions at solar minimum and maximum are shown in Figure 1. At solar minimum, solar wind speed is a strong function of latitude. Thus spacecraft at $0^{\circ}, 3.5^{\circ}$, and $7^{\circ}$ latitude see different solar wind stream structure. At solar maximum, however, the latitudinal structure in solar wind speed is much less prevalent. Consequently, spacecraft at $0^{\circ}, 3.5^{\circ}$, and $7^{\circ}$ latitudes would see very similar solar wind speeds.

We use 1-AU solar wind speed solutions for each Carrington rotation (CR) between 1625 and 2207 (i.e., February 1975-August 2018). For each CR, we extract synthetic spacecraft observations between $\theta=-90^{\circ}$ and $90^{\circ}$ latitude in $1^{\circ}$ steps. At each latitude, $\theta$, the $\mathrm{CR}$ data are combined into a single sequence, $V_{\theta}$, spanning the whole $40+$ years. Differences between the speed at latitude $\theta$ and the heliographic equator, $V_{0}-V_{\theta}$, are computed on a point-by-point basis, i.e. the difference is computed at the same longitude and the same time.

The 1975-2018 interval is also further divided into solar minimum and maximum periods. As in Paper 1, this is done on the basis of solar cycle phase, defined to be 0 and 1 at concurrent solar minima, rather than a sunspot number threshold, as the latter would produce different durations of solar minimum and maximum in small or large sunspot cycles. We define solar minimum and maximum using solar cycle phase limits of 0.17 and 0.67 of the way through the cycle from solar minimum (Owens et al., 2011), which both splits the dataset in half and selects similar sunspot number gradients in the rise and declining phase of each cycle.

As in Paper 1, we note that the model solar wind solutions do not necessarily need to provide accurate forecasts in order to 

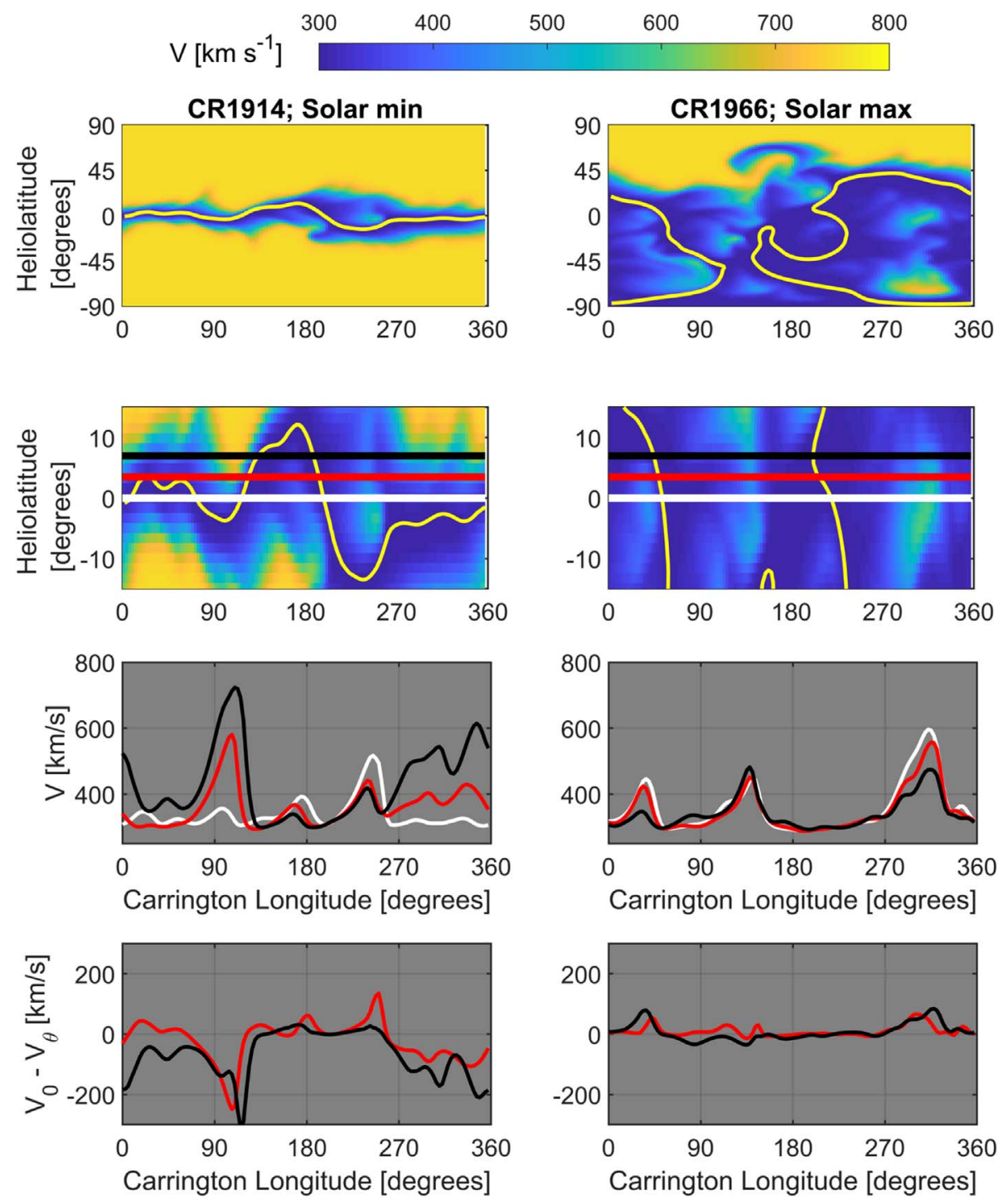

Fig. 1. Examples of model 1-AU solar wind structure at solar minimum (left) and solar maximum (right). Top: solar wind speed at 1 AU as a function of latitude and longitude. The yellow curve shows the heliospheric current sheet. Second row: same as top, but a zoom in of $15^{\circ}$ about the heliographic equator. White, red, and black horizontal lines show $0^{\circ}, 3.5^{\circ}$, and $7^{\circ}$ latitude, respectively. Third row: solar wind speed at the three highlighted latitudes, in the same format. Bottom: the difference in solar wind speed between a given latitude and the equator. Figure adapted from Owens et al. (2019a).

serve the purposes required by this study (following the philosophy expressed by aphorism that "all models are wrong, but some models are useful" Box, 1976). We require only that the model reproduces the solar wind speed structures in a statistical sense. Specifically, we require accurate representation of the latitudinal width and shape of the slow wind band for the given phase of the solar cycle. These features determine the proportions and durations of fast/slow wind at a given latitude. The modelled width of the slow solar wind band has been shown to provide good agreement with the three Ulysses (Wenzel et al., 1992; McComas et al., 2003) latitudinal passes under differing solar activity levels (Jian et al., 2011, 2016; Owens et al., 2017. The proportions of fast and slow wind seen in the ecliptic are generally well reproduced in a point-by-point manner (Owens et al., 2008; Riley et al., 2010; Jian et al., 2015).
More importantly, identification of high-speed streams and allowance for timing errors - which are not critical to the present study - demonstrates that the model produces the general solar wind stream structure near the ecliptic plane (Owens et al., 2005), particularly once transient solar wind structures are discounted (Owens et al., 2008; Jian et al., 2015, 2016). Of course, such timing errors are a critical issue for using such models for forecasting purposes, and it is hoped that the data assimilation of in situ observations enabled by this study will aid in that regard.

\section{Results}

The top-left panel of Figure 2 show the distributions of $V_{\theta}$ as a function of $\theta$. Over the whole 1975-2018 interval, there is a 

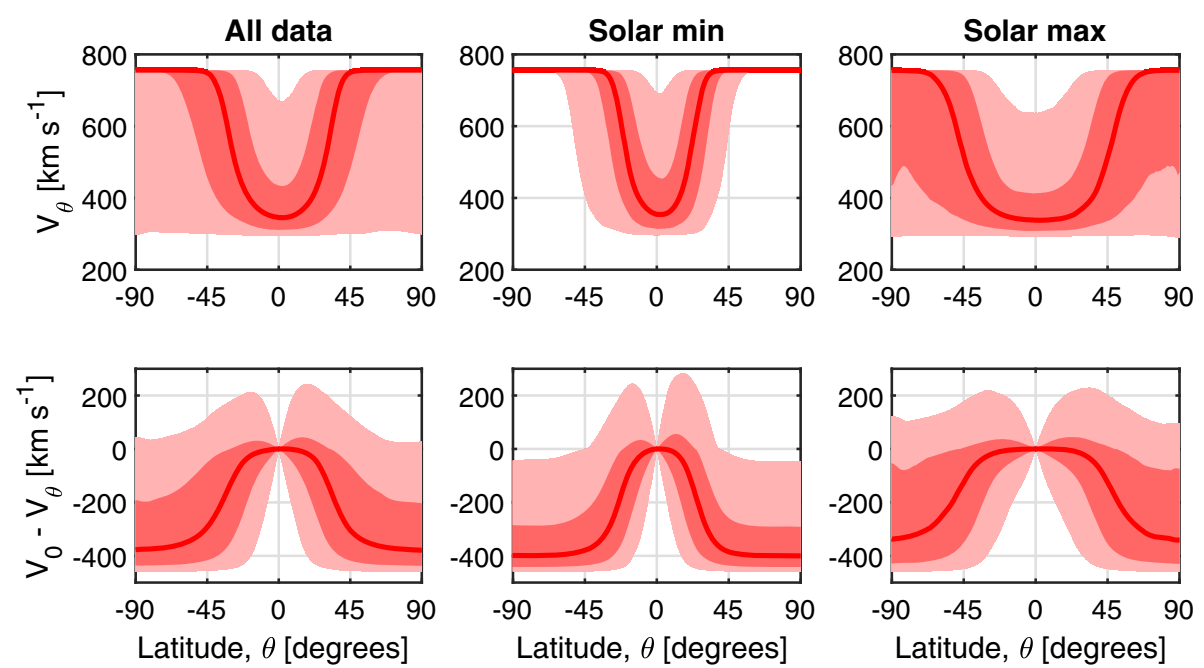

Fig. 2. Top: the distributions of $V_{\theta}$, the solar wind speed at $1 \mathrm{AU}$, as a function of heliographic latitude, $\theta$, for $40+$ years of model results. The red line shows the median, the dark-pink shaded region spans the 25-75 percentiles of the distribution (i.e., the inter-quartile range), while the light-pink regions spans 5-95 percentiles of the $V_{\theta}$ distribution. Panels, from left to right, show the whole dataset, solar minimum, and solar maximum periods, respectively. Bottom: the distributions of the difference in solar wind speed at a given latitude to that at the equator, i.e. $V_{0}-V_{\theta}$, as a function of $\theta$. In the same format as the top panels.

strong tendency for slow wind near the equator and fast wind near the poles, though almost the complete range of speeds can be encountered at all latitudes. The top-centre and top-right panels of Figure 2 show the data further divided into solar minimum and solar maximum conditions, respectively. At solar minimum, latitudes above $60^{\circ}$ show exclusively fast wind, whereas this is not the case at solar maximum, when there exists slow wind even at the poles. Slow solar wind also dominates a broader latitude range about the equator at solar maximum than solar minimum.

The bottom panels of Figure 2 show the distribution of $V_{0}-V_{\theta}$, the speed difference between equator and latitude $\theta$. The median of the $V_{0}-V_{\theta}$ distribution is the bias in assuming $V_{\theta}$ is the same as $V_{0}$. This bias results from different proportions of fast and slow wind at different latitudes. The width of the $V_{0}-V_{\theta}$ distribution is the (random) error in assuming $V_{\theta}$ is the same as $V_{0}$. It results from different longitudinal structure at different latitudes, and hence different phasing in solar wind speed structure.

The variation in $V_{0}-V_{\theta}$ with $\theta$ is fairly complex. Close to the poles, assuming $V_{\theta}$ is representative of $V_{0}$ would introduce a strong negative bias, meaning $V_{0}$ would be overestimated. Similarly, the width of the $V_{0}-V_{\theta}$ distribution is highly asymmetric about the median, and thus the random error would be more likely to lead to large overestimates than large underestimates in $V_{0}$. Closer to the equator, the bias is near zero. The random error decreases towards the equator as well as becoming more symmetric.

As we are primarily interested in latitudes close to the heliographic equator, such as the latitudinal separations of spacecraft in the ecliptic plane, Figure 3 shows the same results confined to within $\pm 10^{\circ}$ latitude. Over this range, the $V_{0}-V_{\theta}$ distribution is centred close to zero, meaning there is little systematic bias. The distribution is also approximately symmetric, with increasing inter-quartile range (and hence random error) with $\theta$. The random error increases far more rapidly with $\theta$ at solar minimum than solar maximum, as expected. This is a result of the latitudinal gradient in solar wind speed near the equator generally being larger at solar minimum than solar maximum. We also note the slight asymmetry between the northern and southern hemispheres. This may be related to uneven sampling of the 22-year Hale cycle, which can affect solar wind properties (e.g., Thomas et al., 2013).

Having surveyed the basic trends in the data, we now quantify the latitudinal representivity properties as a function of latitude and solar activity. This is computed in two forms. Firstly, we compute the latitudinal representivity error introduced by assuming a solar wind speed measurement at a given latitude is representative of the heliographic equator. Secondly, we compute the latitudinal range over which a solar wind speeds can be considered similar, to within a given error tolerance. This determines the latitudinal localisation, i.e. the latitudinal range of influence of a solar wind measurement on the model state.

\subsection{Latitudinal representivity error}

The methodology for determining the latitudinal representivity error (here taken to be the error introduced by assuming a measurement at latitude $\theta$ is representative of the heliographic equator) is demonstrated using $\theta=7^{\circ}$, approximately the largest latitudinal separation from the heliographic equator made by spacecraft in the ecliptic plane. Figure 4 shows the time series of the $V_{0}-V_{7}$ distribution parameters. Different solar cycles have been colour-coded to enable identification of cycleto-cycle variations. The median of $V_{0}-V_{7}$ is close to zero for much of the $40+$ year interval, meaning there is little systematic bias. There are, however, short lived intervals with large biases at the depths of solar minimum. Negative biases, which would mean $V_{0}$ is overestimated on the basis of $V_{7}$ observations, are typically larger than positive biases. The width of the $V_{0}-V_{7}$ distribution, as measured here by the inter-quartile range (IQR), also peaks at solar minimum, but shows a smoother 

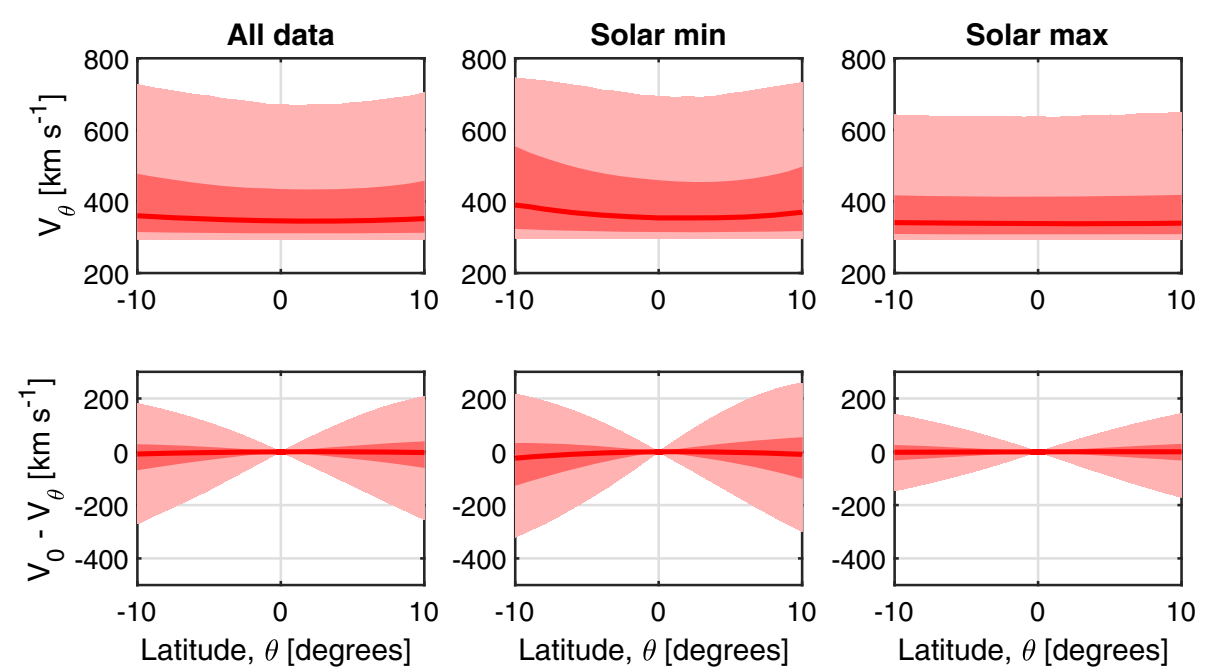

Fig. 3. Same as Figure 2, but only showing latitudes between $-10^{\circ}$ and $10^{\circ}$.
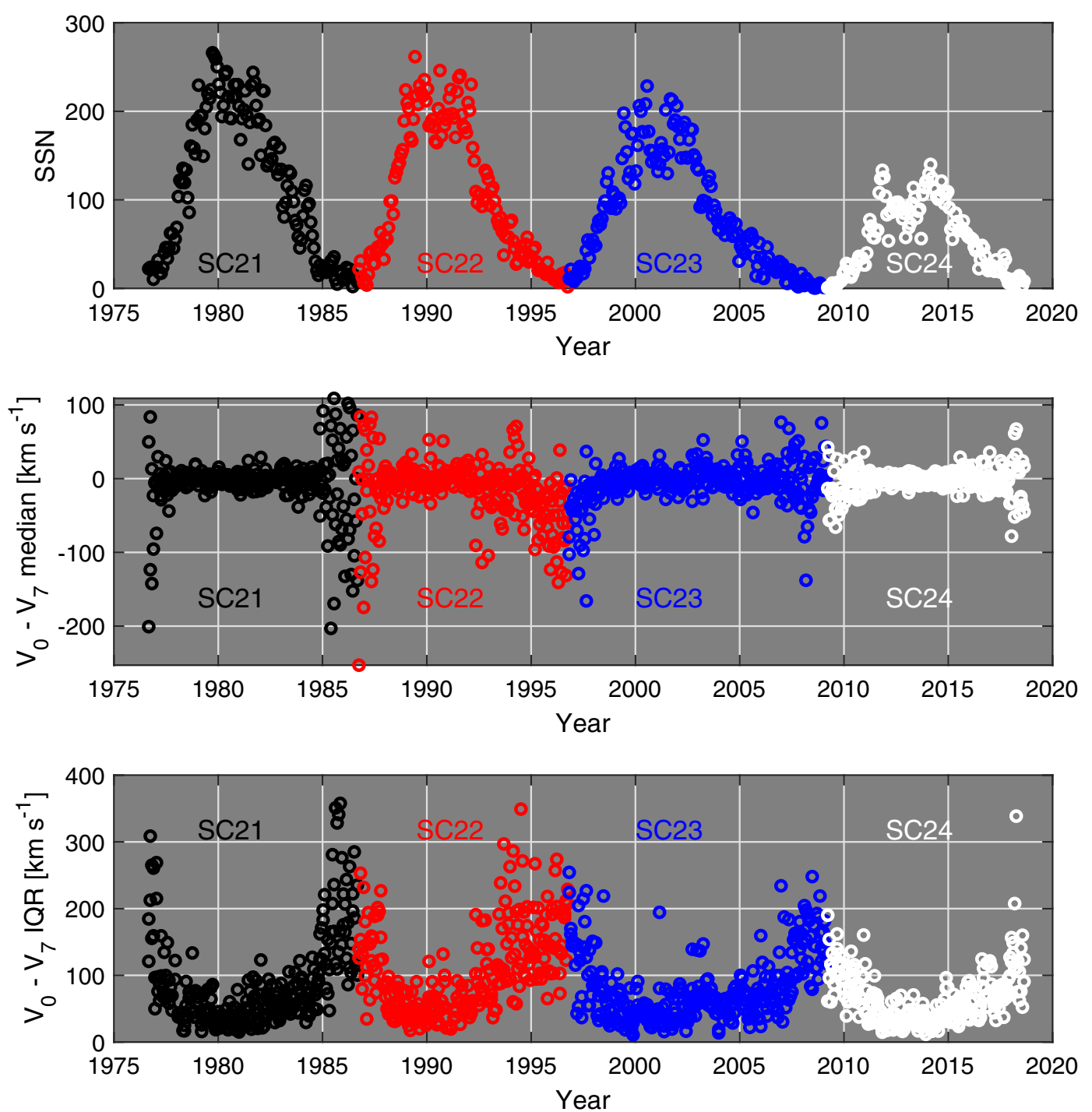

Fig. 4. Time series of 1-CR averages over the period 1975-2018. Solar cycles 21, 22, 23, and 24 have been coloured black, red, blue, and white, respectively. Top: sunspot number. Middle: the median value of $V_{0}-V_{7}$, the solar wind speed difference between the equator and $7^{\circ}$ latitude. Bottom: the inter-quartile range of $V_{0}-V_{7}$. 

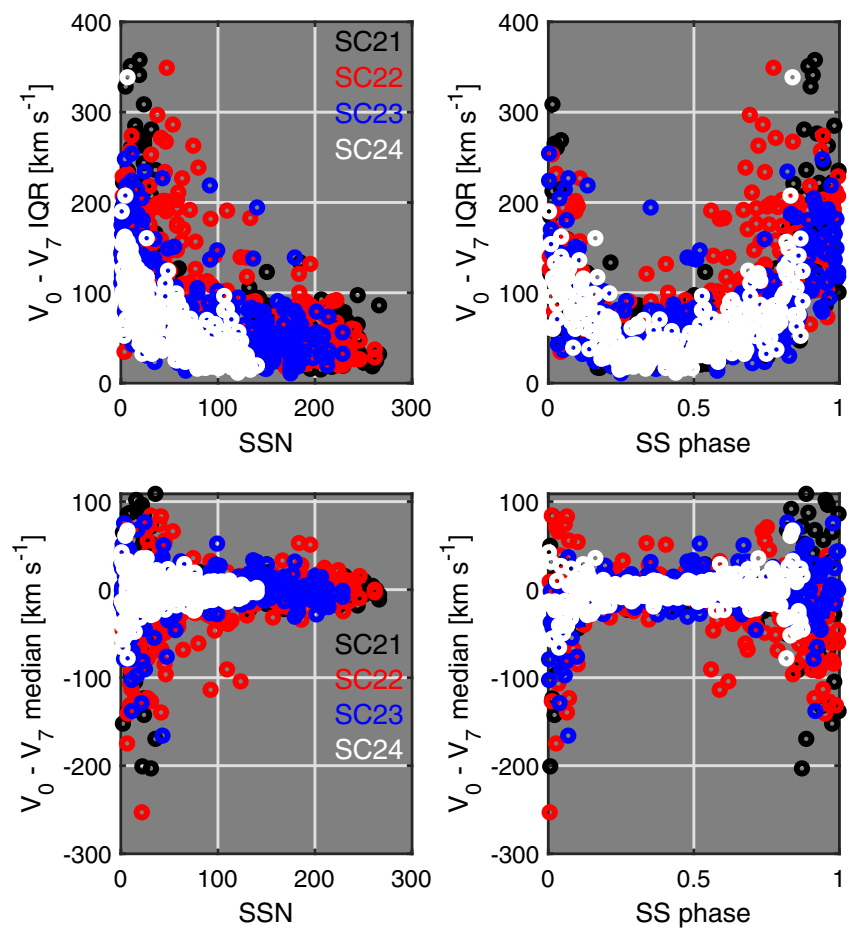

Fig. 5. Scatter plots of $1-\mathrm{CR}$ averages of $V_{0}-V_{7}$, the solar wind speed difference between the equator and $7^{\circ}$ latitude, with solar activity. Top: the inter-quartile range of $V_{0}-V_{7}$ as a function of sunspot number (left) and sunspot cycle phase (right), where solar minimum is 0 and 1 , and solar maximum is approximately 0.5 . Bottom: the median of $V_{0}-V_{7}$ as a function of sunspot number (left) and sunspot cycle phase (right). In all panels, solar cycles 21, 22, 23, and 24 have been coloured black, red, blue, and white, respectively.

variation through the solar cycle. The variation in IQR is asymmetric with regards to the rise and declining phases of the cycle, and the values at the maximum of solar cycle 21 are very similar to those at the maximum of solar cycle 24 , despite the factor 2 decrease in sunspot number. Thus the random latitudinal representivity error is more clearly ordered by solar cycle phase than sunspot number. This is more clearly shown in the scatter plots of Figure 5: when IQR is shown as a function of sunspot number, the four solar cycles separate out, whereas when shown as a function of solar cycle phase, they largely overlap. This behaviour can also be seen to a lesser degree in the median.

Figure 6 shows the joint variation of the $V_{0}-V_{\theta}$ bias (median) and random latitudinal representivity error (IQR or 5-95-percentile range) with latitude and solar cycle phase in equally-spaced bins. The median values are small compared with the IQR values (note the compressed colour scale) at all latitudes and solar cycle phases. The IQR and 5-95 percentile range increase with latitude, as expected, and during the declining phase and solar minimum. These values are listed in Tables 1-3.

\subsection{Latitudinal range of influence}

In the previous section, we computed the latitudinal representivity error, the error introduced by using solar wind measurements to represent speeds at other latitudes. In this section, we determine the inverse; the latitudinal range over which the solar wind can be considered similar, to within some error tolerance. In a three-dimensional solar wind model, this property could be used to inform the localisation volume over which a measurement influences the model state. Thus we refer to this property as the latitudinal range of influence of in situ solar wind measurements as the localisation parameter, $\lambda$.

The methodology for determining $\lambda$ is illustrated in Figure 7 for a hypothetical solar wind observation made at heliographic latitude of $14^{\circ}$ and a solar cycle phase between 0.4 and 0.5 (close to solar maximum). At this latitude and phase of the solar cycle, the median solar wind speed is approximately $340 \mathrm{~km} \mathrm{~s}^{-1}$, with an IQR spanning $310-400 \mathrm{~km} \mathrm{~s}^{-1}$ and the 5 th $\left(95\right.$ th) percentile at $290(610) \mathrm{km} \mathrm{s}^{-1}$. The black lines in the bottom panel show latitudes, $\theta_{\mathrm{MIN}}=-2.7^{\circ}$ and $\theta_{\mathrm{MAX}}=25^{\circ}$, at which either the 5 or 95 percentiles of the $V_{14}-V_{\theta}$ distribution first exceeds the chosen solar wind speed error tolerance, $V_{\mathrm{TOL}}$. (In this example a very large value of $V_{\mathrm{TOL}}=200 \mathrm{~km} \mathrm{~s}^{-1}$ has been used, purely for illustrative purposes.) Thus $\theta_{\mathrm{MIN}}$ and $\theta_{\text {MAX }}$ span the latitudinal range at which at most (i.e., 90\%) of the data points are expected to be within $V_{\mathrm{TOL}}$ of the those at the measurement point. So in this instance, the latitudinal range over which the solar wind can be considered similar to that at $14^{\circ}$ is asymmetric, stretching $16.7^{\circ}$ south of the measurement latitude and $11^{\circ}$ north. As with the $V_{0}-V_{\theta}$ distributions presented in Figure 2, this asymmetry is only noticeable for latitudes greater than approximately $10^{\circ}$. At latitudes associated with the ecliptic plane, $\theta_{\mathrm{MIN}}$ and $\theta_{\mathrm{MAX}}$ are nearly symmetric about the observation latitude. While asymmetric localisation in a DA scheme is possible, we take the simplifying step of defining $\lambda$ to be the smaller of the two latitude ranges, in this instance $11^{\circ}$.

Figure 8 shows $\lambda$ as a function of latitude and solar cycle phase, for a range of $V_{\mathrm{TOL}}$ values. At solar minimum and in the declining phase of the solar cycle, solar wind speed below approximately $40^{\circ}$ is similar over latitudinal ranges smaller than $3^{\circ}$, even when relatively large speed errors are tolerated. Above $50^{\circ}$ the uniform fast wind from the poles means speed is similar for separations above $10^{\circ}$. At solar maximum, the uniform fast wind at the poles is no longer present and the large $\lambda$ at high latitude is also removed. Closer to the equator, $\lambda$ is higher than at solar minimum, so long as moderate to large solar wind speed errors are tolerated.

Restricting analysis to within $7^{\circ}$ of the heliographic equator, the range covered by the ecliptic plane, the variation in $\lambda$ is almost entirely a function of solar cycle with no systematic latitudinal variation. For this reason, Table 4 provides $\lambda$ values averaged over $-7^{\circ}$ to $7^{\circ}$ heliographic latitude, for various $V_{\mathrm{TOL}}$ values and as a function of solar cycle phase.

\section{Other solar wind parameters}

Now that the methodology has been demonstrated for solar wind speed, we repeat the analysis for other solar wind parameters, namely the heliospheric magnetic field (HMF) intensity, $B$, the radial and poloidal components of the $\mathrm{HMF}, B_{r}$ and $B_{p}$ respectively, the proton number density, $n$, and solar wind temperature, $T$. For reasons of space, we only provide the final summary plots here in the main text. Tabulated values are provided in the Supplementary Materials (equivalent to Tables 1-4). 

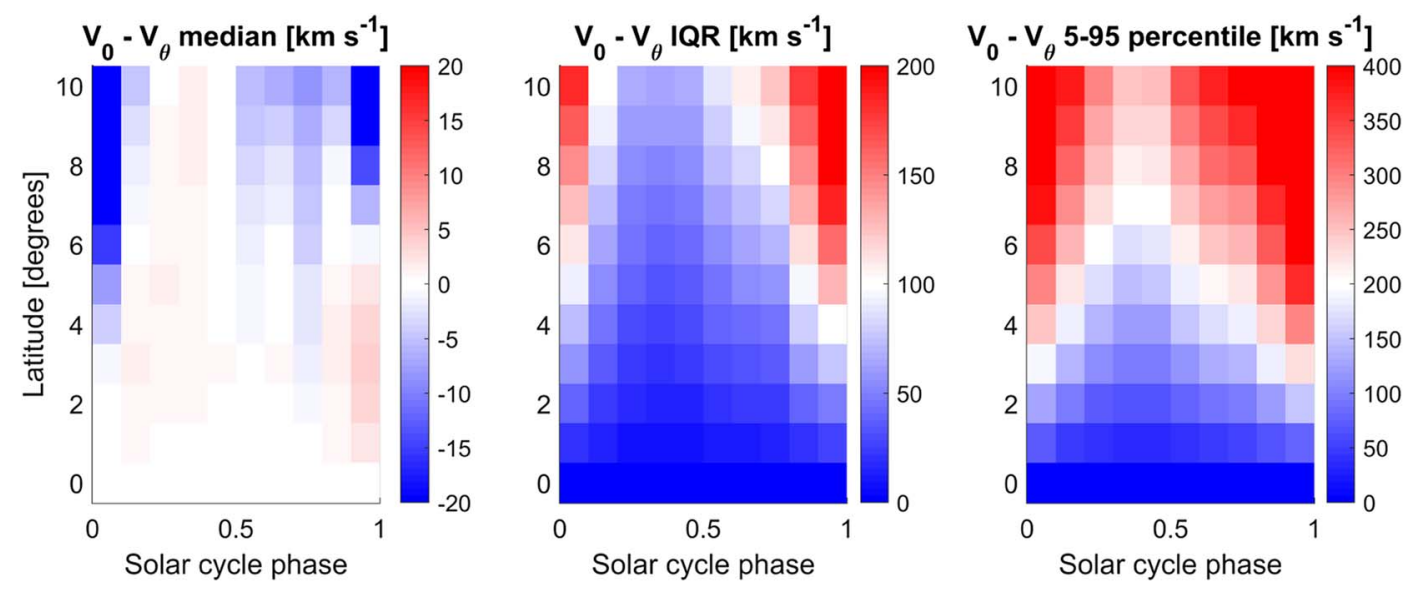

Fig. 6. $V_{0}-V_{\theta}$, solar wind speed difference between the equator and other latitudes, as a function of solar cycle phase and latitude. Left: the median of $V_{0}-V_{\theta}$. Centre: the inter-quartile range of $V_{0}-V_{\theta}$. Right: the 5-95-percentile range of $V_{0}-V_{\theta}$.

Table 1. The median values of the solar wind speed differences between the equator and other latitudes, i.e. $V_{0}-V_{\theta}$, as a function of solar cycle phase and latitude. All values are given in $\mathrm{km} \mathrm{s}^{-1}$.

\begin{tabular}{lrrrrrrrrrr}
\hline Latitude (degrees) & \multicolumn{10}{c}{ Solar cycle phase (bin centre) } \\
\cline { 2 - 9 } & 0.05 & 0.15 & 0.25 & 0.35 & 0.45 & 0.55 & 0.65 & 0.75 & 0.85 \\
\hline 0 & 0.0 & 0.0 & 0.0 & 0.0 & 0.0 & 0.0 & 0.0 & 0.0 & 0.0 & 0.95 \\
1 & 0.3 & 0.5 & 0.2 & 0.2 & 0.2 & -0.3 & 0.2 & -0.6 & 0.5 & 1.7 \\
2 & 0.0 & 0.8 & 0.3 & 0.3 & 0.3 & -0.6 & 0.3 & -1.3 & 0.8 & 3.1 \\
3 & -1.4 & 1.0 & 0.5 & 0.5 & 0.3 & -0.9 & 0.3 & -1.9 & 1.0 & 3.5 \\
4 & -4.4 & 0.7 & 0.8 & 0.6 & 0.2 & -1.1 & 0.1 & -2.4 & 1.0 & 3.1 \\
5 & -8.4 & 0.4 & 0.9 & 0.7 & -0.1 & -1.2 & 0.1 & -2.8 & 0.7 & 2.1 \\
6 & -15.5 & -0.4 & 0.8 & 0.9 & -0.1 & -1.7 & -0.6 & -4.1 & -0.4 & -0.9 \\
7 & -22.8 & -1.3 & 0.9 & 0.9 & 0.1 & -2.5 & -1.6 & -5.1 & -0.8 & -6.3 \\
8 & -31.3 & -2.2 & 0.8 & 1.3 & 0.1 & -3.5 & -2.7 & -5.8 & -1.4 & -14.4 \\
9 & -42.3 & -3.3 & 0.6 & 1.6 & 0.2 & -4.8 & -4.7 & -6.9 & -3.5 & -26.3 \\
10 & -52.1 & -4.7 & 0.1 & 1.3 & -0.5 & -5.7 & -6.8 & -8.7 & -6.3 & -37.1 \\
\hline
\end{tabular}

Table 2. The inter-quartile range of the solar wind speed differences between the equator and other latitudes, i.e. $V_{0}-V_{\theta}$, as a function of solar cycle phase and latitude. All values are given in $\mathrm{km} \mathrm{s}^{-1}$.

\begin{tabular}{|c|c|c|c|c|c|c|c|c|c|c|}
\hline \multirow[t]{2}{*}{ Latitude (degrees) } & \multicolumn{10}{|c|}{ Solar cycle phase (bin centre) } \\
\hline & 0.05 & 0.15 & 0.25 & 0.35 & 0.45 & 0.55 & 0.65 & 0.75 & 0.85 & 0.95 \\
\hline 0 & 0.0 & 0.0 & 0.0 & 0.0 & 0.0 & 0.0 & 0.0 & 0.0 & 0.0 & 0.0 \\
\hline 2 & 37.8 & 22.3 & 14.6 & 12.2 & 13.9 & 18.7 & 20.8 & 23.0 & 38.5 & 48.2 \\
\hline 3 & 55.0 & 33.3 & 22.0 & 18.6 & 21.1 & 27.5 & 30.9 & 33.9 & 58.1 & 73.5 \\
\hline 4 & 73.2 & 43.8 & 29.3 & 25.1 & 28.2 & 36.1 & 41.6 & 45.2 & 77.4 & 100.2 \\
\hline 7 & 126.5 & 72.1 & 48.3 & 45.1 & 48.3 & 61.2 & 72.3 & 82.0 & 130.1 & 185.6 \\
\hline 8 & 144.4 & 80.4 & 54.7 & 51.5 & 54.2 & 71.0 & 81.7 & 95.5 & 144.9 & 216.7 \\
\hline 9 & 162.5 & 89.2 & 60.8 & 58.2 & 59.6 & 79.5 & 93.1 & 108.2 & 160.5 & 245.6 \\
\hline 10 & 181.7 & 98.1 & 66.8 & 63.8 & 65.2 & 88.4 & 104.8 & 120.7 & 176.2 & 269.7 \\
\hline
\end{tabular}

Figures 9-13 show that other solar wind parameters follow the same general pattern of behaviour as solar wind speed. That is, the error in assuming a measurement at latitude $\theta$ is representative of the helioequator, $X_{0}-X_{\theta}$, grows far more rapidly with $\theta$ during solar minimum than during solar maximum. Accordingly, $\lambda$, the range of latitudes over which an observation is representative to within some error tolerance, also increases toward solar maxmimum and is minimum at solar minimum. 
Table 3. The 5-to-95-percentile range of the solar wind speed differences between the equator and other latitudes, i.e. $V_{0}-V_{\theta}$, as a function of solar cycle phase and latitude. All values are given in $\mathrm{km} \mathrm{s}^{-1}$.

\begin{tabular}{lrrrrrrrrrr}
\hline Latitude (degrees) & \multicolumn{10}{c}{ Solar cycle phase (bin centre) } \\
\cline { 2 - 9 } & 0.05 & 0.15 & 0.25 & 0.35 & 0.45 & 0.55 & 0.65 & 0.75 & 0.85 & 0.95 \\
\hline 0 & 0.0 & 0.0 & 0.0 & 0.0 & 0.0 & 0.0 & 0.0 & 0.0 & 0.0 & 0.0 \\
1 & 65.7 & 46.8 & 36.4 & 30.1 & 30.9 & 38.9 & 45.0 & 47.2 & 62.4 & 77.0 \\
2 & 127.8 & 92.5 & 71.5 & 60.1 & 61.0 & 76.2 & 87.9 & 92.4 & 121.6 & 150.8 \\
3 & 188.4 & 136.9 & 106.2 & 90.9 & 91.3 & 113.2 & 128.3 & 137.8 & 180.6 & 224.3 \\
4 & 241.0 & 178.8 & 139.3 & 120.3 & 118.9 & 148.5 & 165.9 & 179.1 & 230.5 & 291.4 \\
5 & 296.0 & 220.4 & 169.5 & 146.6 & 149.3 & 184.3 & 206.0 & 219.9 & 281.2 & 360.6 \\
6 & 337.8 & 254.0 & 198.4 & 171.2 & 173.3 & 213.1 & 242.7 & 254.0 & 323.7 & 415.2 \\
7 & 381.1 & 288.7 & 224.3 & 194.1 & 194.9 & 244.8 & 277.7 & 289.2 & 365.3 & 467.6 \\
8 & 421.4 & 321.2 & 249.1 & 212.8 & 216.3 & 271.7 & 311.4 & 322.2 & 401.2 & 509.7 \\
9 & 449.2 & 347.9 & 270.3 & 228.4 & 233.2 & 300.3 & 340.2 & 359.6 & 430.2 & 545.2 \\
10 & 477.4 & 373.0 & 292.6 & 246.8 & 248.7 & 330.5 & 370.1 & 395.5 & 457.3 & 581.1 \\
\hline
\end{tabular}
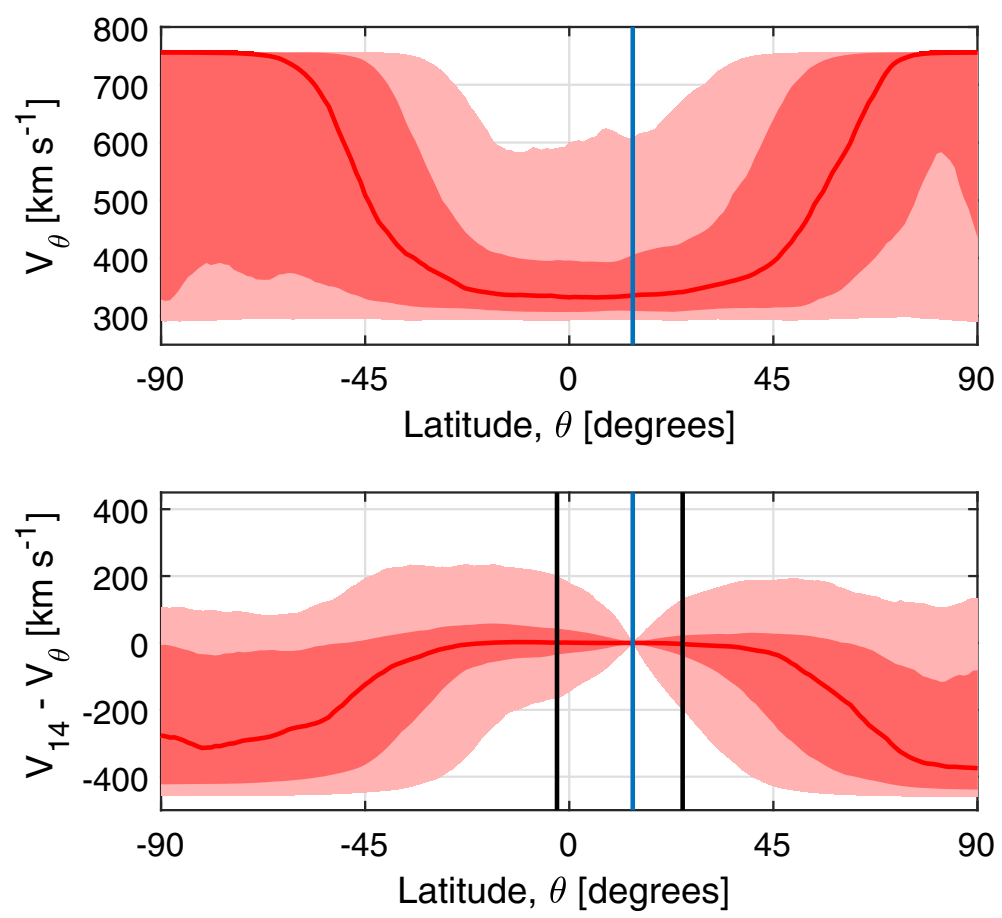

Fig. 7. Determining the localisation parameter, $\lambda$, at a latitude of $14^{\circ}$, the vertical blue line. Top: the distribution of solar wind speeds as a function of latitude for an interval close to solar maximum (solar cycle phase between 0.4 and 0.5 ). The red line shows the median, the darkpink shaded region spans the 25-75 percentiles of the distribution (i.e., the inter-quartile range), while the light-pink regions spans 5-95 percentiles. Bottom: the distribution of $V_{14}-V_{\theta}$, speed differences between $14^{\circ}$ latitude and $\theta$. The vertical black lines show the latitudes where either the 5 or 95 percentiles of the $V_{14}-V_{\theta}$ distribution first exceed $V_{\mathrm{TOL}}=200 \mathrm{~km} \mathrm{~s}^{-1}$ in magnitude.

There is little variation in $\lambda$ with the latitude of observation, at least over the range $\pm 10^{\circ}$ about the helioequator.

While these qualitative trends are expected to be robust, the quantitative values are unlikely to be as reliable as the solar wind speed. The magnetic field intensity, and hence individual HMF components, is known to be underestimated by coronal models (Owens et al., 2008; Stevens et al., 2012). There are similar biases in density and temperature. Thus we expect the $X_{0}-X_{\theta}$ values to represent an lower bound on the true values, and the $\lambda$ to be an upper bound.

\section{Summary and conclusions}

Data assimilation (DA) is the combination of model and observational information, taking account of the uncertainties in both. In order to effectively utilize in situ solar wind speed observations in solar wind models, it is necessary to quantify the latitudinal range over which measurements are representative. Available solar wind observations themselves are insufficient for this purpose. Instead we have utilised $40+$ years of data-driven global solar wind model results. The model 

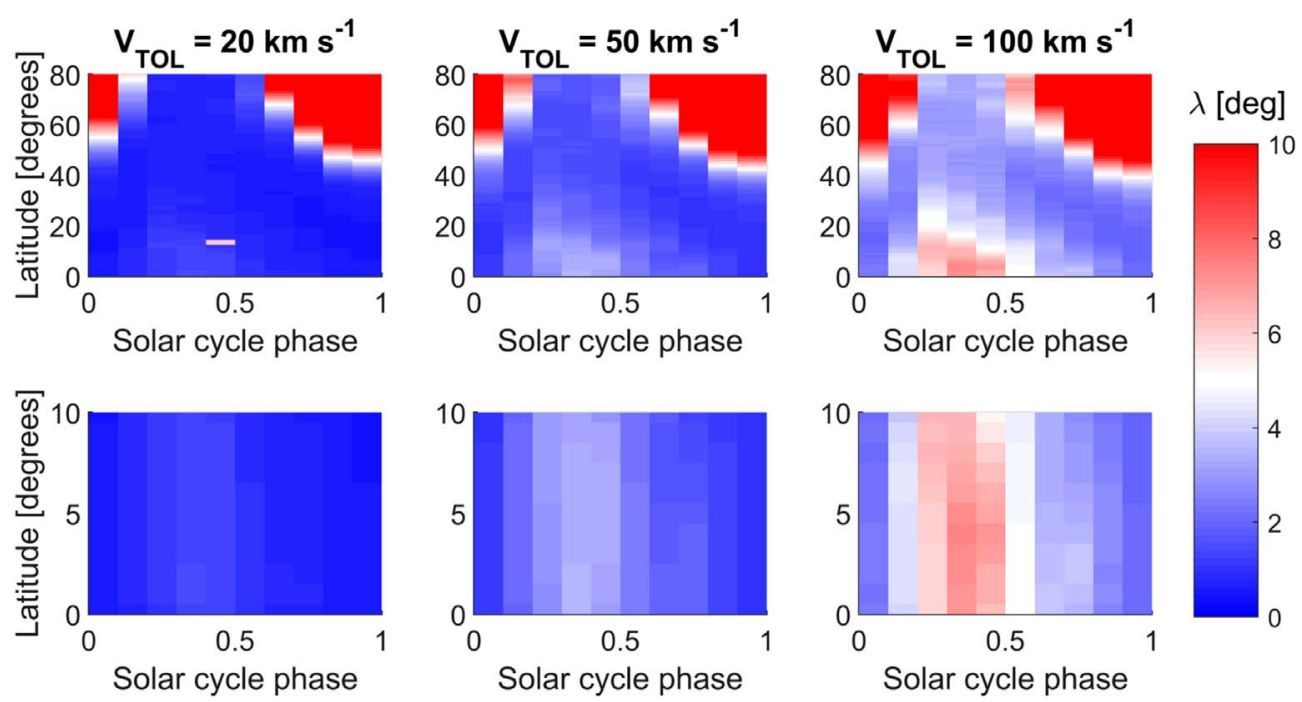

Fig. 8. The localisation parameter, $\lambda$, in degrees, as a function of latitude and solar cycle phase. Note the colour bar has been saturated at $10^{\circ}$ to highlight the variations in smaller values. Top: the full latitude range. Bottom: a detail of $\pm 10^{\circ}$. Columns, from left to right, show speed error tolerances of $V_{\mathrm{TOL}}=20,50$, and $100 \mathrm{~km} \mathrm{~s}^{-1}$, respectively.

Table 4. $\lambda$ for solar wind near the in-ecliptic plane (averaged over $\pm 7^{\circ}$ ), in degrees, as a function of solar cycle phase and $V_{\mathrm{TOL}}$.

\begin{tabular}{|c|c|c|c|c|c|c|c|c|c|c|}
\hline$V_{\mathrm{TOL}}\left(\mathrm{km} \mathrm{s}^{-1}\right)$ & \multicolumn{10}{|c|}{ Solar cycle phase (bin centre) } \\
\hline 10 & 0.2 & 0.4 & 0.5 & 0.6 & 0.6 & 0.4 & 0.3 & 0.3 & 0.2 & 0.2 \\
\hline 30 & 0.7 & 1.2 & 1.7 & 2.0 & 1.9 & 1.4 & 1.0 & 1.0 & 0.7 & 0.6 \\
\hline 40 & 0.9 & 1.6 & 2.2 & 2.6 & 2.5 & 1.9 & 1.4 & 1.4 & 1.0 & 0.8 \\
\hline 50 & 1.1 & 2.0 & 2.8 & 3.4 & 3.2 & 2.3 & 1.7 & 1.7 & 1.3 & 1.0 \\
\hline 150 & 3.5 & 6.7 & 10.1 & 11.1 & 10.3 & 7.6 & 5.3 & 5.1 & 3.9 & 3.1 \\
\hline 200 & 4.8 & 9.3 & 16.2 & 15.3 & 13.7 & 10.5 & 7.2 & 6.8 & 5.4 & 4.3 \\
\hline
\end{tabular}

solutions were used to compute two related aspects of solar wind latitudinal representivity. Firstly, the latitudinal representivity error introduced by using solar wind speed observations to represent latitudes away from the point of measurement. Specifically, we have quantified the latitudinal representivity error introduced by assuming a measurement at a given latitude can be used to represent the value at the heliographic equator. This property would form part of the observational error in using measurments away from the heliographic equator in a two-dimensional DA scheme. Secondly, we quantified the latitudinal range over which the solar wind can be considered to be similar (to within some given speed error tolerance). This informs the latitudinal range over which a measurement should influence the model state in a three-dimensional DA scheme, known as the measurment localisation.

For the latitudinal representivity error, we compute both the systematic bias and random error in assuming the solar wind speed measured at a latitude $\theta, V_{\theta}$, is representative of the speed at the heliographic equator, $V_{0}$. As $\theta$ increases (either towards the north or south pole), there is increasing systematic bias that leads to a large overestimate in $V_{0}$. This effect is most pronounced at solar minimum, particularly above $45^{\circ}$ latitude. For spacecraft in the ecliptic plane, however, the latitudinal separation from the equator is limited to $\pm 7.5^{\circ}$. Within this limited range, the bias is essentially zero. Thus for data assimilation of in-ecliptic solar wind observations, only the random latitudinal representivity error need be considered. For in-ecliptic measurements, the random error increases linearly with $\theta$, but is also a strong function of solar activity level. We have provided a look-up table of this representivity error as a function of latitude and phase of the solar cycle.

The trends are similar in the second measure, $\lambda$, the latitudinal range over which solar wind speed can be considered similar and thus should influence the model state. Near the ecliptic plane, $\lambda$ is only one or two degrees at solar minimum and during the declining phase of the solar cycle, even when relatively large solar wind speed errors are tolerated. This is in broad agreement with the findings of Paper 1 regarding the inherent uncertainty in L5 observations for providing a forecast of near-Earth solar wind via simple corotation. 

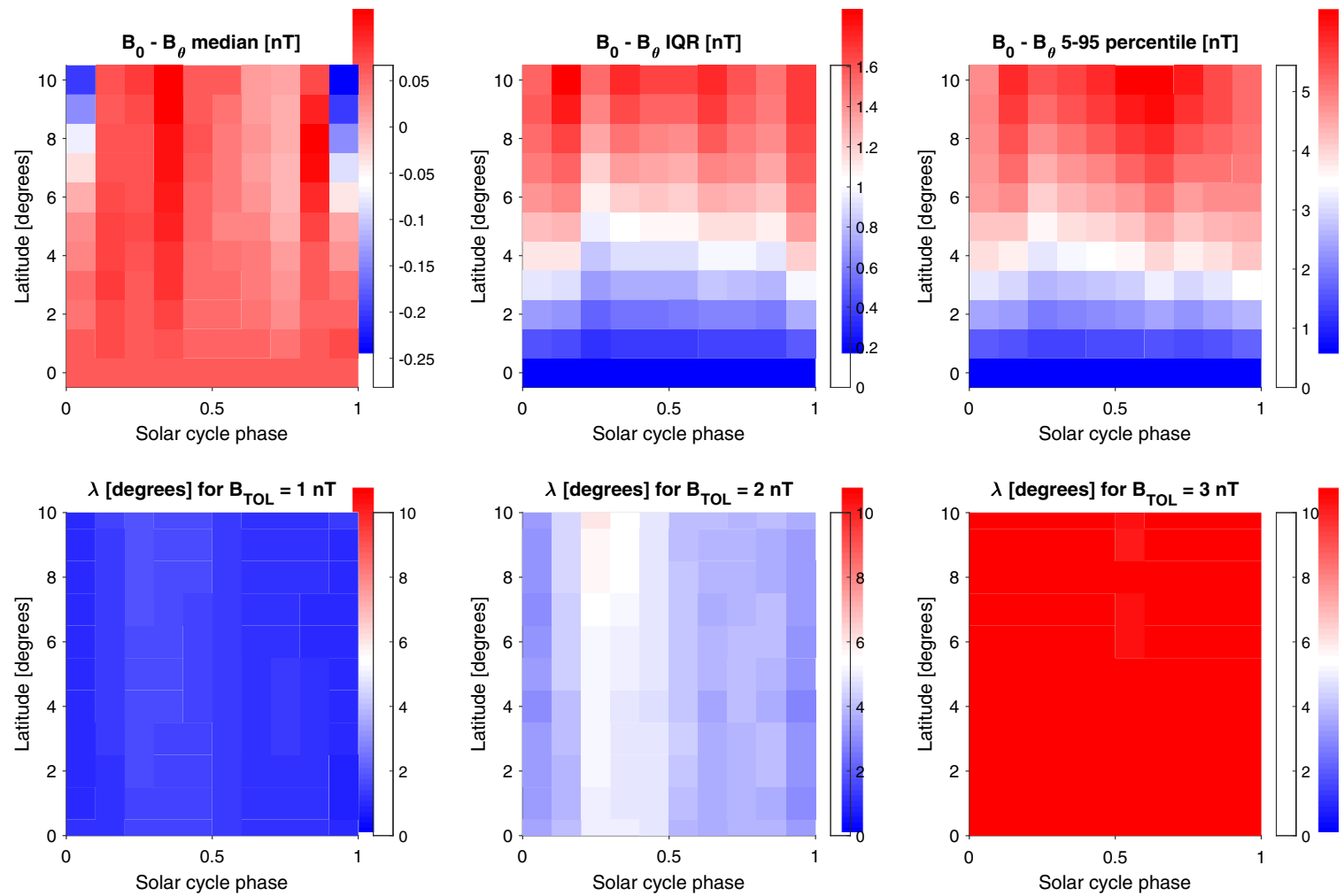

Fig. 9. Summary plots for the HMF intensity, $B$. Top: Figure 6 , but for $B$. That is, the error introduced by assuming $B$ at latitude $\theta$ is representative of latitude 0 , i.e. $B_{0}-B_{\theta}$. Panels, from left to right show the median, interquartile range, and 5-95 percentile range of the distributions as a function of latitude and solar cycle phase. Bottom: Figure 8, but for $B$. That is, the localisation parameter, $\lambda$, as a function of latitude and solar cycle for three $B$ error tolerances of $B_{\mathrm{TOL}}=1,2$, and $3 \mathrm{nT}$.
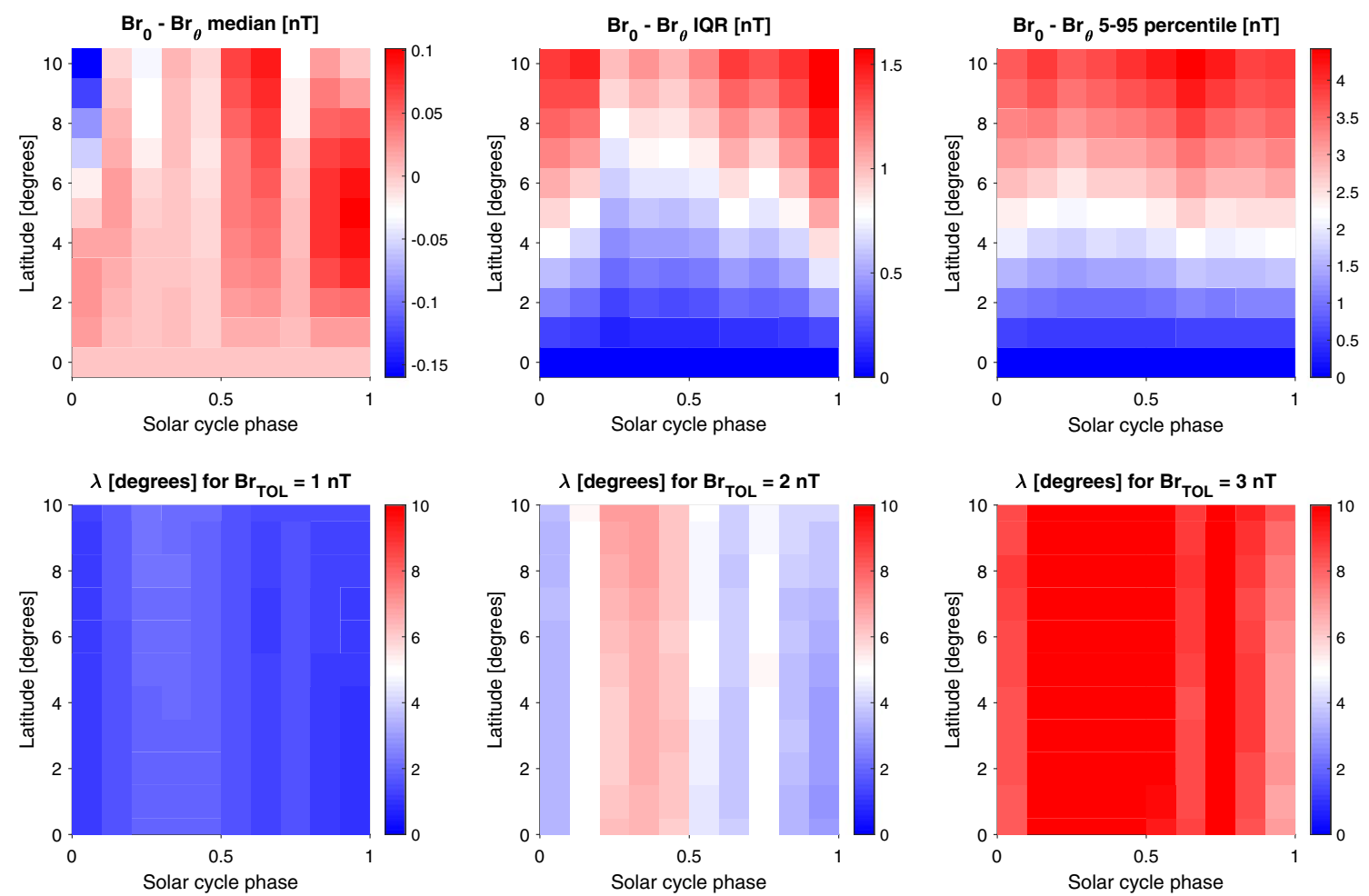

Fig. 10. Summary plots for the radial HMF component, $B_{r}$, in the same format as Figure 9. 

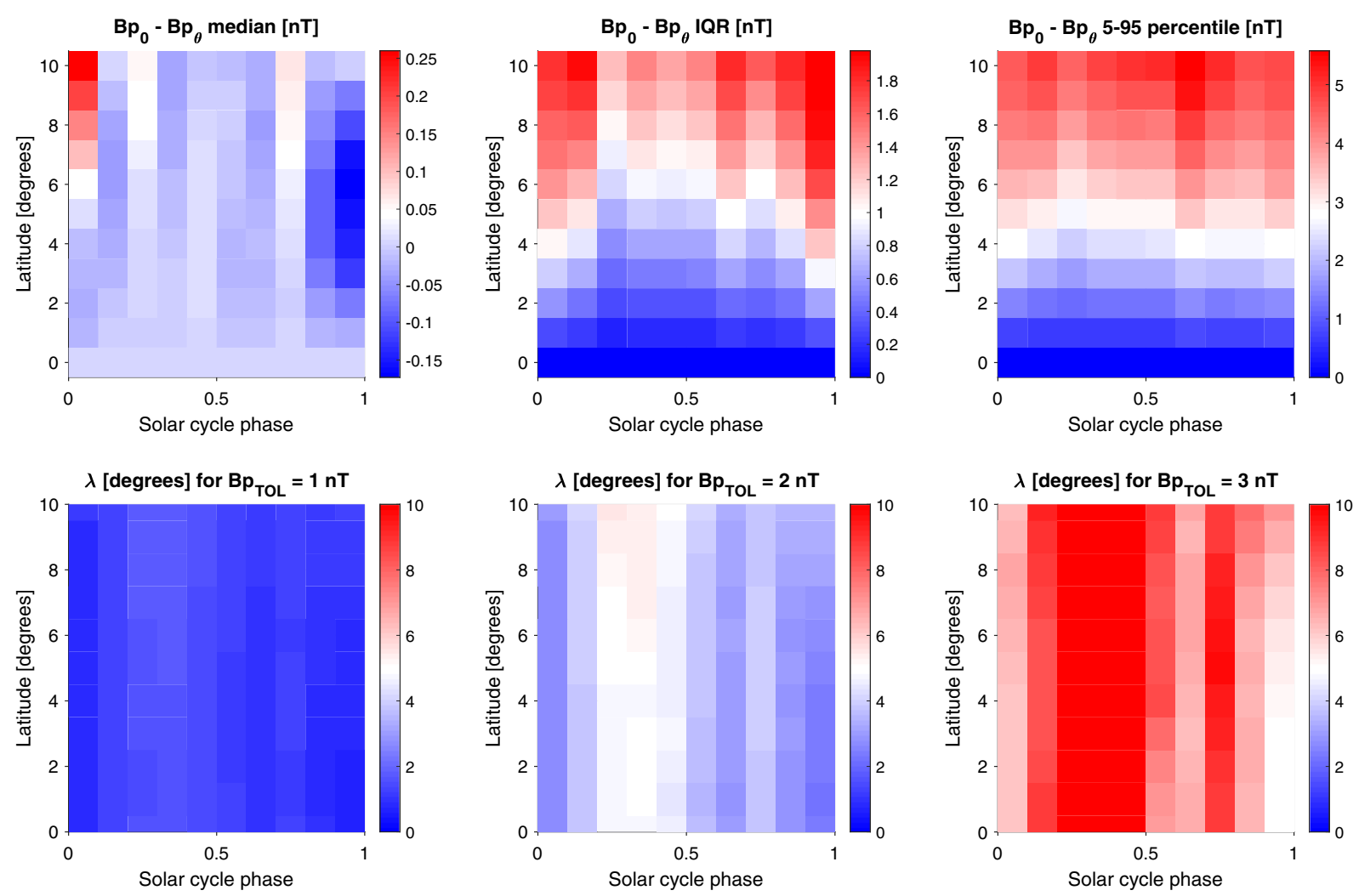

Fig. 11. Summary plots for the poloidal HMF component, $B_{p}$, in the same format as Figure 9.
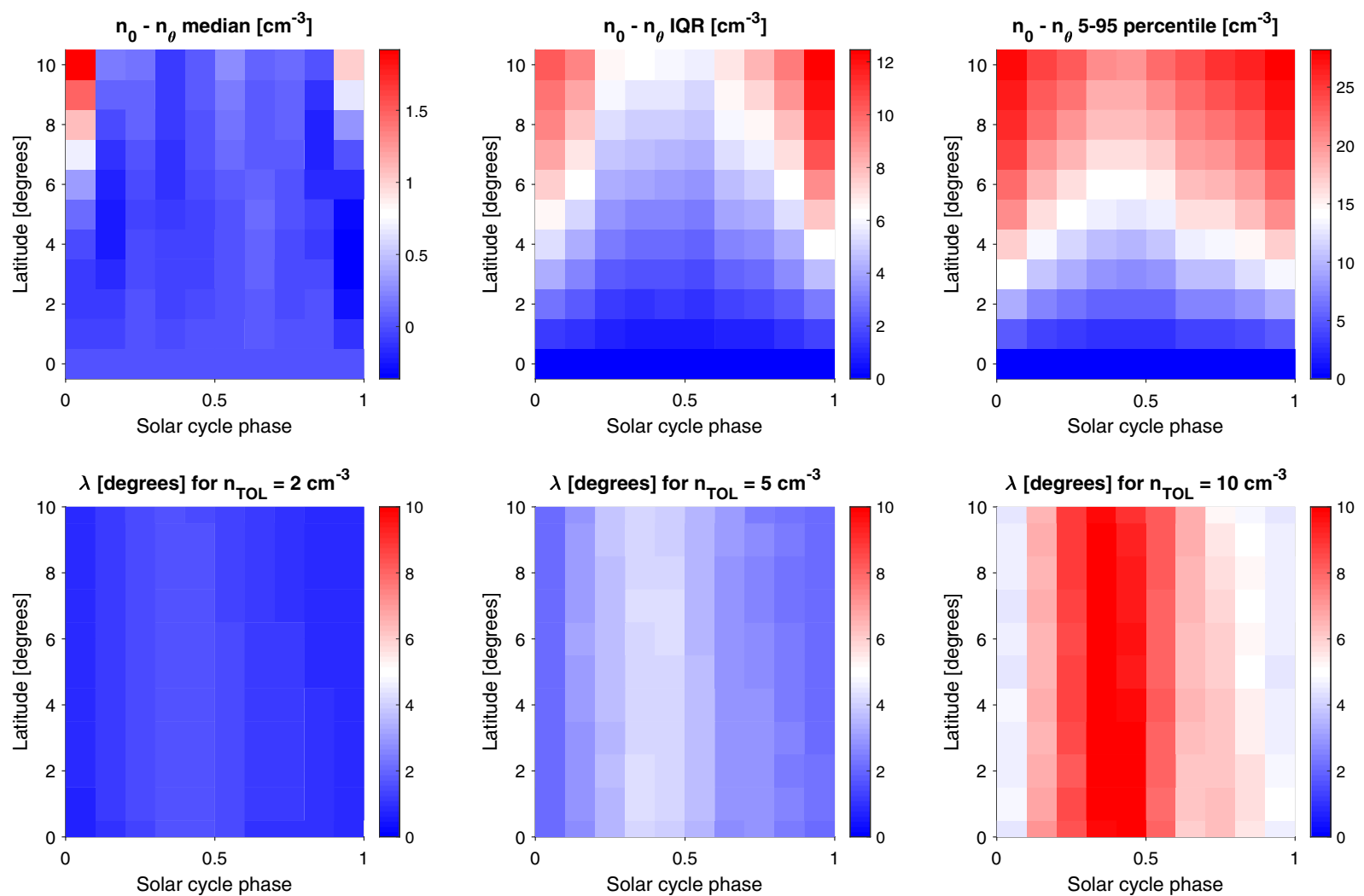

Fig. 12. Summary plots for the proton number density, $n$, in the same format as Figure 9. 

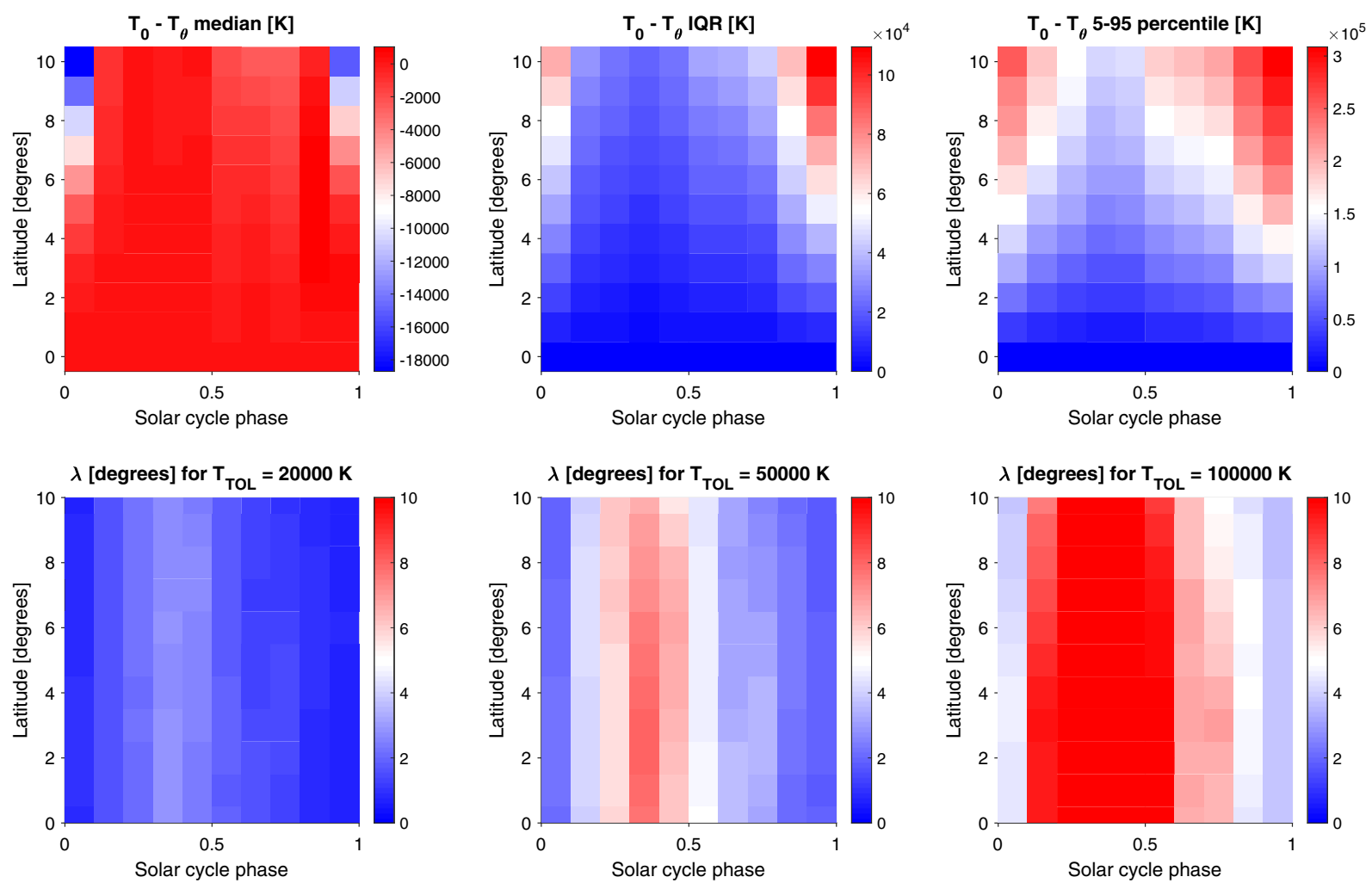

Fig. 13. Summary plots for the solar wind temperature, $T$, in the same format as Figure 9.

At solar maximum, $\lambda$ rises to approximately $5^{\circ}$ for large error tolerances.

We note that the solar wind model used in this study assumes that the solar wind is perfectly steady-state over a solar rotation. It therefore does not routinely capture transient solar wind structures, such as those resulting from coronal mass ejections. And it does not capture small-scale structure resulting from solar wind turbulence. Thus the model variability results only from large-scale coronal structure and should be regarded as a lower-limit of real measurements. Hence the respresentivity error is an lower limit and the latitudinal range of influence is an upper limit. Furthermore, the results presented here apply to spatial scales greater than the model grid size $\left(\sim 10^{6} \mathrm{~km}\right.$ at $\left.1 \mathrm{AU}\right)$ and time scales greater than the associated time scale of approximately $1 \mathrm{~h}$.

Looking beyond solar wind speed, we provided estimates of the representivity error and the latitudinal range of representivity for the heliospheric magnetic field intensity and individual components, the plasma density and temperature. The qualitative trends closely follow those found for solar wind speed, as expected. Given the known systematic biases in the model estimates of these other solar wind properties, we do not expect the quantitative values to be as accurate as solar wind speed. However, they should provide a useful starting point from which to build.

In future work, we will utilise the values presented here in the assimilation of in situ solar wind observations with both two- and three-dimensional solar wind models. But the current results suggest that in-ecliptic in situ observations have strong limitations in terms of usefulness for solar wind DA. Thus while emote sensing observations likely present significantly higher measurement errors than in situ observations, they may nevertheless be invaluable for providing adequate spatial sampling for reliable solar wind forecasting.

\section{Supplementary materials}

Supplementary material to the below is available at https:// www.swsc-journal.org/10.1051/swsc/2020009/olm

Each sheet contains information for a different physical parameter.

Rows 6 to 17 describe the latitudinal respresentivity error. That is, the error (in units of the physical parameter) introduced by assuming a measurement at latitude theta is representative of the value at the heliographic equator.

Values are given for different latitudes and solar cycle phases. The median, interquartile and 9 to 95 percentile ranges are given.

Rows 22 to 31 give the latitudinal range of influence of a measurement (in degrees), for various error tolerance levels. Values have been averaged over -7 to +7 latitude.

Acknowledgements. This Work was part-funded by Science and Technology Facilities Council (STFC) grant numbers ST/M000885/1 and ST/R000921/1, and Natural Environment Research Council (NERC) grant number NE/P016928/1. PR gratefully acknowledges support from NASA (80NSSC18K0100 and NNX16AG86G) and NOAA (NA18NWS4680081). The heliospheric MAS solutions used 
in this study can be visualised at http://www.predsci.com/ mhdweb/plot_2d.php and downloaded at http://www.predsci. com/mhdweb/data_access.php. The editor thanks two anonymous reviewers for their assistance in evaluating this paper.

\section{References}

Arge CN, Odstrcil D, Pizzo VJ, Mayer LR. 2003. Improved method for specifying solar wind speed near the sun. AIP Conf Proc 679 (1): 190-193. https://doi.org/10.1063/1.1618574.

Asch M, Bocquet M, Nodet M. 2016. Data assimilation: Methods, algorithms, and applications, Vol. 11 of Fundamentals of Algorithms, SIAM, Philadelphia.

Aseev N, Shprits Y. 2019. Reanalysis of ring current electron phase space sensities using Van Allen Probe observations, convection model, and lognormal Kalman filter. Space Weather 17(4): 619638. https://doi.org/10.1029/2018SW002110.

Barnard L, Owens M, Scott C, Jones S. 2019. Extracting innerheliosphere solar wind speed information from heliospheric imager observations. Space Weather 17(6): 925-938. https://doi.org/ 10.1029/2019SW002226.

Box GEP. 1976. Science and statistics. J Am Stat Assoc 71(356): 791-799. https://doi.org/10.1080/01621459.1976.10480949.

Bust GS, Mitchell CN. 2008. History, current state, and future directions of ionospheric imaging. Rev Geophys 46(1): RG1003. https://doi.org/10.1029/2006RG000212.

Elvidge S, Angling MJ. 2019. Using the local ensemble transform Kalman filter for upper atmospheric modelling. J Space Weather Space Clim 9: A30. https://doi.org/10.1051/swsc/2019018.

Ghil M, Malanotte-Rizzoli P. 1991. Data assimilation in meteorology and oceanography. Adv Geophys 33: 141-266. https://doi.org/ 10.1016/S0065-2687(08)60442-2.

Glauert SA, Horne RB, Meredith NP. 2018. A 30-year simulation of the outer electron radiation belt. Space Weather 16(10): 14981522. https://doi.org10.1029/2018SW001981.

Hickmann KS, Godinez HC, Henney CJ, Arge CN. 2015. Data assimilation in the ADAPT photospheric flux transport model. Sol Phys 290(4): 1105-1118. https://doi.org/10.1007/s11207-015-0666-3.

Janji T, Bormann N, Bocquet M, Carton JA, Cohn SE, et al. 2018. On the representation error in data assimilation. Quart J Roy Meteor Soc 144(713): 1257-1278. https://doi.org/10.1002/qj.3130.

Jian LK, Russell CT, Luhmann JG, MacNeice PJ, Odstrcil D, Riley P, Linker JA, Skoug RM, Steinberg JT. 2011. Comparison of observations at ACE and Ulysses with Enlil model results: Stream interaction regions during Carrington rotations 2016-2018. Sol Phys 273(1): 179-203. https://doi.org/10.1007/s11207-011-9858-7.

Jian LK, MacNeice PJ, Taktakishvili A, Odstrcil D, Jackson B, Yu H-S, Riley P, Sokolov IV, Evans RM. 2015. Validation for solar wind prediction at Earth: Comparison of coronal and heliospheric models installed at the CCMC. Space Weather 13(5): 316-338. https://doi.org/10.1002/2015SW001174.

Jian LK, MacNeice PJ, Mays ML, Taktakishvili A, Odstrcil D, Jackson B, Yu H-S, Riley P, Sokolov IV. 2016. Validation for global solar wind prediction using Ulysses comparison: Multiple coronal and heliospheric models installed at the Community Coordinated Modeling Center. Space Weather 14(8): 592-611. https://doi.org/10.1002/2016SW001435.

Kahler SW, Arge CN, Smith DA. 2016. Using the WSA model to test the Parker spiral approximation for SEP event magnetic connections. Sol Phys 291(6): 1829-1852. https://doi.org/10.1007/ s11207-016-0934-x.
Kalnay E. 2002. Atmospheric modeling, data assimilation and predictability. Cambridge University Press, Cambridge.

Lang M, Owens MJ. 2019. A variational approach to data assimilation in the solar wind. Space Weather 17(1): 59-83. https://doi.org/10.1029/2018SW001857.

Lang M, Browne P, van Leeuwen PJ, Owens M. 2017. Data assimilation in the solar wind: Challenges and first results. Space Weather 15(11): 1490-1510. https://doi.org/10.1002/2017SW001681.

Linker J, Mikic Z, Biesecker DA, Forsyth RJ, Gibson WE, Lazarus AJ, Lecinski A, Riley P, Szabo A, Thompson BJ. 1999. Magnetohydrodynamic modeling of the solar corona during whole sun month. J Geophys Res 104: 9809-9830. https://doi.org/ 10.1029/1998JA900159.

MacNeice P, Jian LK, Antiochos SK, Arge CN, Bussy-Virat CD, et al. 2018. Assessing the quality of models of the ambient solar wind. Space Weather 16(11): 1644-1667. https://doi.org/10.1029/ 2018SW002040.

Manoharan PK. 2012. Three-dimensional evolution of solar wind during solar cycles 22-24. Astrophys J 751(2): 128-141. https://doi.org/10.1088/0004-637X/751/2/128.

Mays ML, Taktakishvili A, Pulkkinen A, MacNeice PJ, Rastätter L, et al. 2015. Ensemble modeling of CMEs using the WSAENLIL + Cone model. Sol Phys 290(6): 1775-1814. https://doi.org/10.1007/ s11207-015-0692-1.

McComas DJ, Elliott HA, Schwadron NA, Gosling JT, Skoug RM, Goldstein BE. 2003. The three-dimensional solar wind around solar maximum. Geophys Res Lett 30(10): 1517. https://doi.org/ 10.1029/2003GL017136.

McGregor SL, Hughes WJ, Arge CN, Owens MJ, Odstrcil D. 2011. The distribution of solar wind speeds during solar minimum: Calibration for numerical solar wind modeling constraints on the source of the slow solar wind. J Geophys Res 116(A3): 1-11. https://doi.org/10.1029/2010JA015881.

Odstrcil D. 2003. Modeling 3-D solar wind structures. Adv Space Res 32: 497-506. https://doi.org/10.1016/S0273-1177(03)00332-6.

Owens MJ, Arge CN, Spence HE, Pembroke A. 2005. An eventbased approach to validating solar wind speed predictions: Highspeed enhancements in the Wang-Sheeley-Arge model. J Geophys Res 110(A12): 1-10. https://doi.org/10.1029/2005JA011343.

Owens MJ, Spence HE, McGregor S, Hughes WJ, Quinn JM, Arge CN, Riley P, Linker J, Odstrcil D. 2008. Metrics for solar wind prediction models: Comparison of empirical, hybrid, and physicsbased schemes with 8 years of L1 observations. Space Weather 6(8): S08,001. https://doi.org/10.1029/2007SW000380.

Owens MJ, Lockwood M, Barnard L, Davis CJ. 2011. Solar cycle 24: Implications for energetic particles and long-term space climate change. Geophys Res Lett 38(19): 1-5. https://doi.org/ 10.1029/2011GL049328.

Owens MJ, Lockwood M, Riley P. 2017. Global solar wind variations over the last four centuries. Sci Rep 7(41): 548. https://doi.org/10.1038/srep41548.

Owens M, Riley P, Lang M, Lockwood M. 2019a. Near-earth solar wind forecasting using corotation from L5: The error introduced by heliographic latitude offset. Space Weather 17(7): 11051113.

Owens MJ, Lang M, Riley P, Stansby D. 2019b. Towards construction of a solar wind reanalysis dataset: Application to the first perihelion pass of Parker Solar Probe. Sol Phys 294(6): 83. https://doi.org/10.1007/s11207-019-1479-6.

Pomoell J, Poedts S. 2018. EUHFORIA: European heliospheric forecasting information asset. J Space Weather Space Clim 8: A35. https://doi.org/10.1051/swsc/2018020. 
Rabier F. 2005. Overview of global data assimilation developments in numerical weather-prediction centres. Quart J Roy Meteor Soc 131(613): 3215-3233. https://doi.org/10.1256/qj.05.129.

Riley P, Linker JA, Mikic Z. 2001. An empirically-driven global MHD model of the solar corona and inner heliosphere. J Geophys Res 106: 15889-15902. https://doi.org/10.1029/2000JA000121.

Riley P, Luhmann J, Opitz A, Linker JA, Mikic Z. 2010. Interpretation of the cross-correlation function of ACE and STEREO solar wind velocities using a global MHD Model. J Geophys Res Space Phys 115(A11): 104. https://doi.org/10.1029/2010JA015717.

Riley P, Linker JA, Lionello R, Mikic Z. 2012. Corotating interaction regions during the recent solar minimum: The power and limitations of global MHD modeling. J Atmos Sol-Terr Phys 83: 1-10. https://doi.org/10.1016/j.jastp.2011.12.013.

Riley P, Linker JA, Arge CN. 2015. On the role played by magnetic expansion factor in the prediction of solar wind speed. Space Weather 13(3): 154-169. https://doi.org/10.1002/2014SW001144.

Sasaki Y. 1970. Numerical variation analysis formulated under the constraints as determined by longwave equations and a low-pass filter. Mon Weather Rev 98(12): 884-898. https://doi.org/10.1175/ 1520-0493(1970).

Stevens ML, Linker JA, Riley P, Hughes WJ. 2012. Underestimates of magnetic flux in coupled MHD model solar wind solutions.
J Atmos Sol-Terr Phys 83: 22-31. https://doi.org/10.1016/ j.jastp.2012.02.005.

Temmer M, Hinterreiter J, Reiss MA. 2018. Coronal hole evolution from multi-viewpoint data as input for a STEREO solar wind speed persistence model. J Space Weather Space Clim 8: A18. https://doi.org/10.1051/swsc/2018007.

Thomas SR, Owens MJ, Lockwood M. 2013. The 22-year hale cycle in cosmic ray flux - evidence for direct heliospheric modulation. Sol Phys 289(1): 407-421. https://doi.org/10.1007/s11207-013-0341-5.

Toth G, Sokolov IV, Gombosi TI, Chesney DR, Clauer CR, et al. 2005. Space weather modeling framework: A new tool for the space science community. J Geophys Res 110(A12): 226. https://doi.org/10.1029/2005JA011126.

Waller JA, Dance SL, Lawless AS, Nichols NK, Eyre JR. 2014. Representativity error for temperature and humidity using the Met Office high-resolution model. Quart J Roy Meteor Soc 140(681): 1189-1197. https://doi.org/10.1002/qj.2207.

Wenzel KP, Marsden RG, Page DE, Smith EJ. 1992. The ULYSSES mission. A\&A Suppl 92(2): 207-219.

Zhao X-P, Plunkett SP, Liu W. 2002. Determination of geometrical and kinematical properties of halo coronal mass ejections using the cone model. J Geophys Res 107(A8): SSH-13. https://doi.org/ 10.1029/2001JA009143.

Cite this article as: Owens MJ, Lang M, Riley P, Lockwood M \& Lawless AS. 2020. Quantifying the latitudinal representivity of in situ solar wind observations. J. Space Weather Space Clim. 10, 8. 